\title{
ISOLATION AND CHARACTERIZATION OF SOME FRAGMENTS OBTAINED AFTER PEPTIC DIGESTION OF BOVINE SERUM ALBUMIN
}

\author{
W. G. M. Braam, B. J. M. Harmsen, J. A. L. I. Walters and G. A. J. van Os \\ Department of Biophysical Chemistry, University of Nijmegen, \\ Nijmegen, The Netherlands
}

Received 25 March 1971

\begin{abstract}
Bovine serum albumin was hydrolysed by pepsin at pH 3.7. Ten well-defined fragments were found, as was shown by electrophoresis on polyacrylamide gels at $p H 3.0$ in the presence of $6 M$ urea. Three of the fragments could be isolated by precipitation with trichloroacetic acid followed by ion exchange chromatography. The procedure is suited to obtain large quantities in a simple and reproducible way.

The fragments were identified by polyacrylamide gel electrophoresis, terminal amino acid determination, and amino acid analysis. The largest of the three fragments appeared to be composed of the two other fragments and is probably placed C-terminal in albumin. Some other properties of the largest fragment were also determined, e.g., the a-helix content and the number of titratable residues.
\end{abstract}

In a previous paper we described the hydrolysis of bovine serum albumin by pepsin in the $\mathrm{pH}$ region of the NF (Normal-Fast) transition (1).

According to Foster (2), this transition can be xplained by assuming that the single polypeptide chain of albumin consists of four compact units. In the normal form, present around the isoelectric point, these units are close together; but upon lowering the $\mathrm{pH}$, the distance between the units increases as a consequence of electrostatic repulsion, although they still remain linked by short randomly coiled peptide chains. This results in the appearance of a form with a higher electrophoretic mobility (fast form), because protons are bound when the intrasurfaces between the units come in contact with the solvent. In this situation an enzyme with a low specificity, such as pepsin, has been suggested to attack preferentially the links between the compact units (3). So, in order to ob- tain the units, digestion of albumin can best be carried out at a $\mathrm{pH}$ value at which the NF transition is complete but further partial unfolding of the units has not yet occurred.

If there are four units (three sites of hydrolysis), then 10 particles can be expected upon digestion. Indeed digestion at $\mathrm{pH}$ values between 3.6 and 3.9 revealed ten well-defined fragments as observed by polyacrylamide gel electrophoresis (1).

Because of the close relationship between the hydrolysis of albumin by pepsin and the NF transition, these fragments may have an important structural function in the albumin molecule. For an analysis of the albumin molecule, in terms of its composing parts, the isolation and characterization of the fragments is of value. This paper deals with three of these fragments which could be isolated in a simple and reproducible way. 


\section{EXPERIMENTAL}

Bovine serum albumin was obtained from Nutritional Biochemicals Corp. $(4 \times$ crystallized; lot no. 8161) or from Poviet Producten N.V., Amsterdam, The Netherlands (lot no. 399). No differences were found between these samples. After blocking the SH group, the incubation with pepsin was performed for $30 \mathrm{~min}$. at $\mathrm{pH} 3.7$ as described earlier (1). The digest was examined by means of electrophoresis on polyacrylamide gels in $6 \mathrm{M}$ urea at $\mathrm{pH} 3.0$.

A prefractionation of the digest was obtained by means of precipitation by trichloroacetic acid (TCA) at $0^{\circ}$. The digest was diluted with water to a protein concentration of $3 \%$. An equal volume of a $4 \%$ solution of TCA was added slowly under continuous stirring. After one hour the precipitate was removed by centrifugation at $10,000 \mathrm{~g}$. To the supernatant solution, TCA was added to a final concentration of $10 \%$. After one hour the mixture was centrifugated at $10,000 \mathrm{~g}$ and the precipitate was solved in $6 \mathrm{M}$ urea $(\mathrm{pH}$ 3.0), also containing $0.1 \mathrm{M} \mathrm{NaCl}$.

Ion exchange chromatography was performed at $4^{\circ}$ on SE Sephadex C-50. The smaller Sephadex particles were removed by decantation. The yield of $50 \mathrm{~g}$ albumin, digested and prefractionated as described above, was applied to a column with gel-bed dimensions $45 \times 240 \mathrm{~mm}$. After elution of the column with $500 \mathrm{ml} 6 \mathrm{M}$ urea $(0.1 \mathrm{M} \mathrm{NaCl}$, formic acid to $\mathrm{pH} 3.0$ ), the solution of the precipitate found in $10 \%$ TCA was applied to the column and eluated with $500 \mathrm{ml}$ portions of a solution containing $6 \mathrm{M}$ urea $(\mathrm{pH} 3.0)$ and increasing amounts of $\mathrm{NaCl}(0.15 \mathrm{M}, 0.22 \mathrm{M}$, $0.26 \mathrm{M}, 0.30 \mathrm{M}$ ). The fractions were desalted on Sephadex G-25 at pH $2.8(\mathrm{HCl})$. Finally the samples were lyophylized and stored in vacuum at $4^{\circ}$ in the presence of $\mathrm{P}_{2} \mathrm{O}_{5}$.

Amino acid analysis was performed on an automatic amino acid analyser (Technicon) using a $130 \mathrm{~cm}$ column. Hydrolysis with $\mathrm{HCl}$ (Suprapur, Merck, Darmstadt) was carried out at $105^{\circ}$ $\pm 0.5^{\circ}$ for 2448 hours. From each fragment at least three independently isolated samples were examined. Each sample was analysed on two separate columns.

N-terminal amino acids were determined as DNP-amino acids according to the procedure given by Bloemendal (4). The DNP-amino acids were identified by thin layer chromatography on silica gel plates (F 254, Merck, Darmstadt) as described by Brenner, Niederweiser, and Pataki (5). Better results were obtained when the systems 3 and 4 of these authors were replaced by the following solutions: chloroform/tertiary butyl alcohol/acetic acid $(60+40+3 \mathrm{vol})$ and benzene/ pyridine/acetic acid $(50+50+2 \mathrm{vol})$ respectively.

$C$-terminal amino acid analysis was carried out as described by Peters, Logan, and Sanford (6). Carboxypeptidase A (DFP treated; $3 \times$ crystallized) was obtained from Serva. The ratio carboxypeptidase:fragment was about 1:400 (w/w). After various incubation times, samples were taken and norleucine was added as an internal standard. The amino acids were determined quantitatively on an automatic amino acid analyzer (Technicon). To remove an amide which had nearly the same reference value as threonine, the mixture was heated in $1 \mathrm{~N} \mathrm{HCl}$ for one hour at $100^{\circ}$ before analysis. Differential hydrogen ion titration curves were determined as described by De Bruin and Van Os (7) and Janssen, De Bruin, and Van Os (8). The protein concentration was about $1 \%$. The titrations were performed in $0.1 \mathrm{M}$ $\mathrm{KCl}$.

The optical rotation was measured with a Jasco spectropolarimeter model ORD/CD/UV-5, equipped with a $450 \mathrm{~W}$ Xenon lamp. A cell with a path length of $10 \mathrm{~mm}$ was used. The changes in optical rotation at $313 \mathrm{~m} \mu$ are usually small. Therefore, for measurements at this wavelength, the cell containing the protein solution was placed in series with a cell filled with a solution of sucrose of such a concentration that the rotation of the sample solution was nearly compensated. In this way it was possible to increase the sensitivity of the spectropolarimeter in order to enhance the accuracy of the readings. Protein concentrations varied from 0.02 to $0.8 \%$ and all solutions contained $0.1 \mathrm{M} \mathrm{KCl}$.

Molecular weights were found by means of equilibrium centrifugation with a model $\mathbf{E}$ Spinco ultracentrifuge equipped with an electronic recording system for absorption optics as described by Van Es and Bont (9).

\section{RESULTS AND DISCUSSION}

Fig. 1 shows the ten fragments obtained after peptic digestion of albumin. In order to isolate some of these fragments, it appeared to be desirable to carry out a preseparation during which only a few fragments are removed from the mixture of ten. This was done by precipitation with 


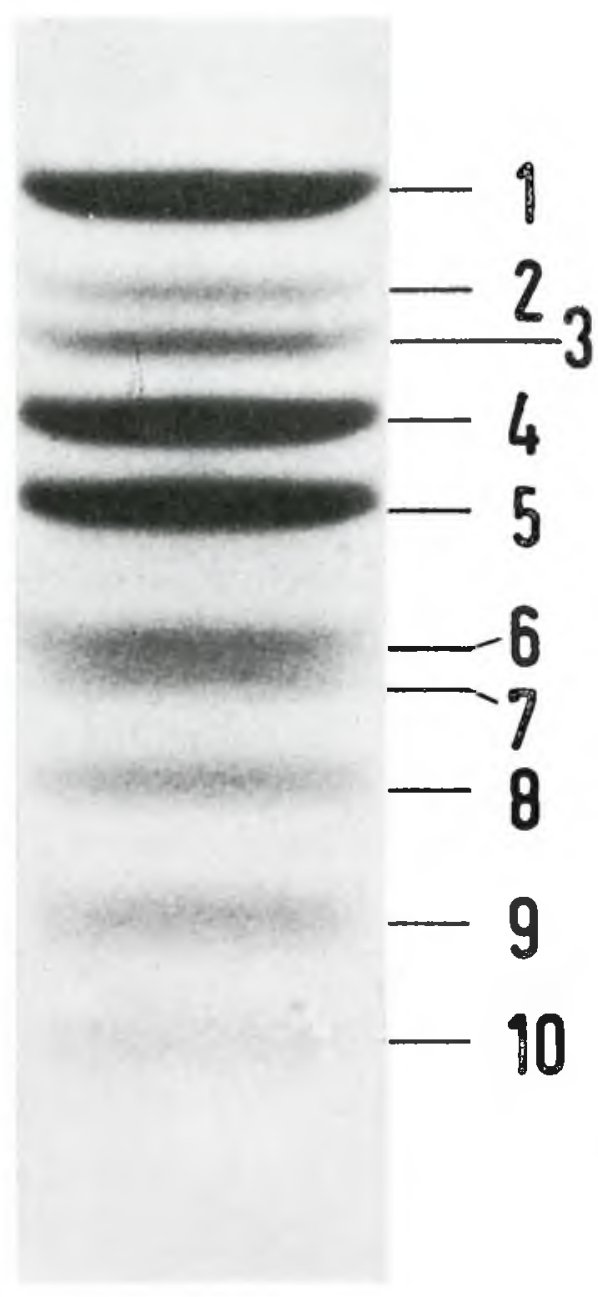

FIGURE 1

Polyacrylamide gel electrophoresis at pH 3.0 in $6 \mathrm{M}$ urea after incubation of albumin with pepsin at $\mathrm{pH}$ -7 for $30 \mathrm{~min}$. The arrow indicates the direction of iigration.

TCA according to Peters and Hawn (10). Other methods, as for example salting out with $\left(\mathrm{NH}_{4}\right)_{2} \mathrm{SO}_{4}$ at $\mathrm{pH} 2.5$, were not successful. Precipitation by TCA also seems attractive in view of the results of Schwert (11) who found that albumin recovered after this precipitation was identical to the untreated protein with respect to sedimentation, electrophoretic behaviour, solubility, and crystallizibility. At concentrations from 1.8 to $2.2 \%$ TCA, all protein components in the solution were precipitated completely, except three fragments (see Fig. 2, tube 1). At lower concentrations small amounts of other fragments also remained in solution. The three fragments could be separated by discontinuous chromatography on SE Sephadex C-50 at pH 3.0. Addition of urea was necessary to prevent aggregation. Fig. 3 shows the elution pattern obtained in this

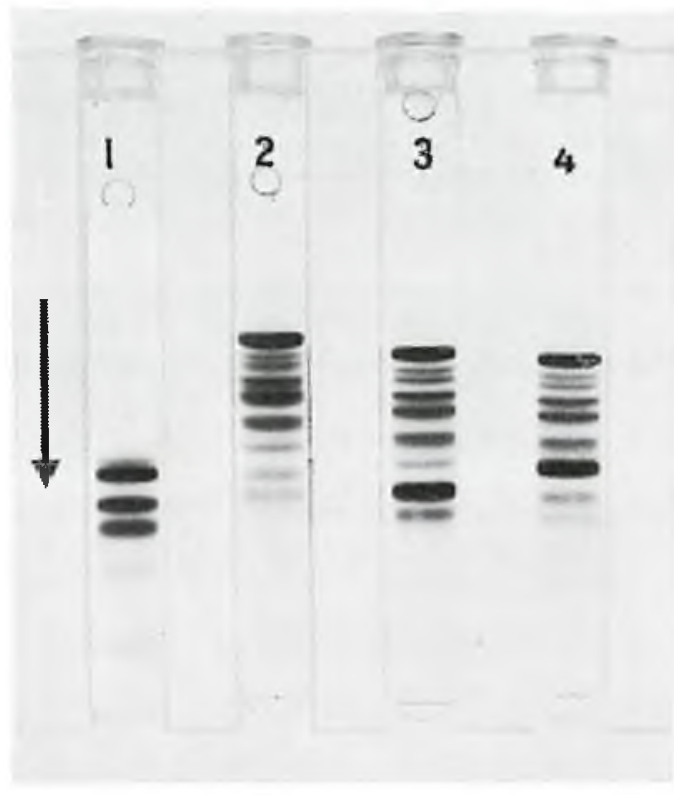

Figure 2

Polyacrylamide gel electrophoresis at pH 3.0 in $6 \mathrm{M}$ urea. The arrow indicates the direction of migration. (1) Fraction of the digest which precipitates between $2 \%$ and $10 \%$ TCA. (2) Peptic digest obtained after incubation of albumin at $\mathrm{pH} 3.7$ for $30 \mathrm{~min}$ (see Fig. 1). (3) The same digest with fragment 9 added. (4) The same with fragment 8 added.

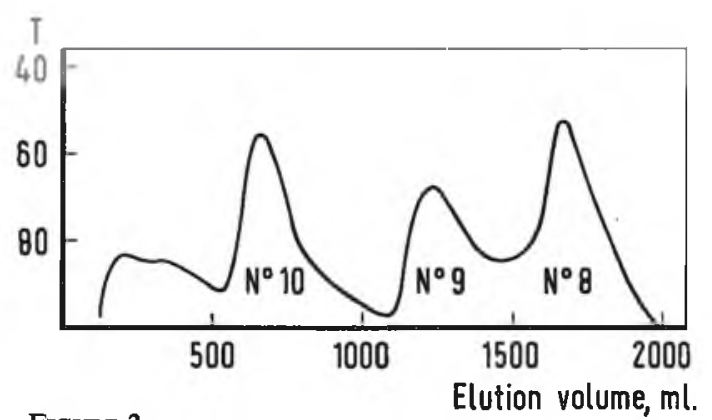

FIGURE 3

Elution pattern of the TCA fraction on SE Sephadex $\mathrm{C}-50$ at $\mathrm{pH} 3.0$ in the presence of $6 \mathrm{M}$ urea. The transmittance at $254 \mathrm{~m} \mu$ was measured as a function of the elution volume. The elution was discontinuous with successively $0.16,0.22,0.26$, and $0.30 \mathrm{M} \mathrm{NaCl}$. 
way. The first peak appeared to be heterogeneous. The next peaks each contained only a single protein component as was shown by electrophoresis on polyacrylamide gels. This procedure was repeated many times and was found to be completely reproducible. It was necessary to keep the time of incubation short $(30 \mathrm{~min})$ as already mentioned by Braunitzer, Hilschmann, and Müller (12). Probably the small active pepsin fragments, products of the autolysis of the enzyme, do have a somewhat different specificity. In a few cases the fragments were not pure enough but could be purified further by gel-filtration on Sephadex G-50 or G-75.

The fragments were identified by gel electrophoresis of a mixture containing the purified fragment and a sufficient amount of the total digestion mixture (Fig. 2). In this way it was found that the fragments 8,9 , and 10 had been isolated.

Because fragment 8 was found to disappear after prolonged incubation, it was treated once more with pepsin (Fig. 4). As a result of this incubation two fragments were obtained which were identical to 9 and 10 on polyacrylamide gel electrophoresis. This suggests that $\mathbf{8}$ is composed of 9 and 10.

For the molecular weight of 10,9 , and 8 , we found $3,000,12,000$, and 15,000 respectively, assuming a partial specific volume of 0.73 which is equal to that of albumin itself.

Table 1 shows the amino acid composition of the fragments. If 8 is composed of 9 and 10 , then the amino acid composition of 8 should be the same as that of 9 and 10 together. As can be seen from Table 1 the agreement is rather good, although there remain some small differences. Table 1 also contains the results of the terminal amino acid determinations. The $\mathrm{N}$-terminal amino acid of both 8 and 9 appeared to be valine. The thin layer patterns of the fragments 8 and: were remarkably identical. Only traces of impurities were found. Therefore we can conclude that 9 is $\mathrm{N}$-terminal and 10 is C-terminal in 8. For fragment 8,-Leu-Ala $\mathrm{OH}$ was found as Cterminal sequence which is the same as in albumin

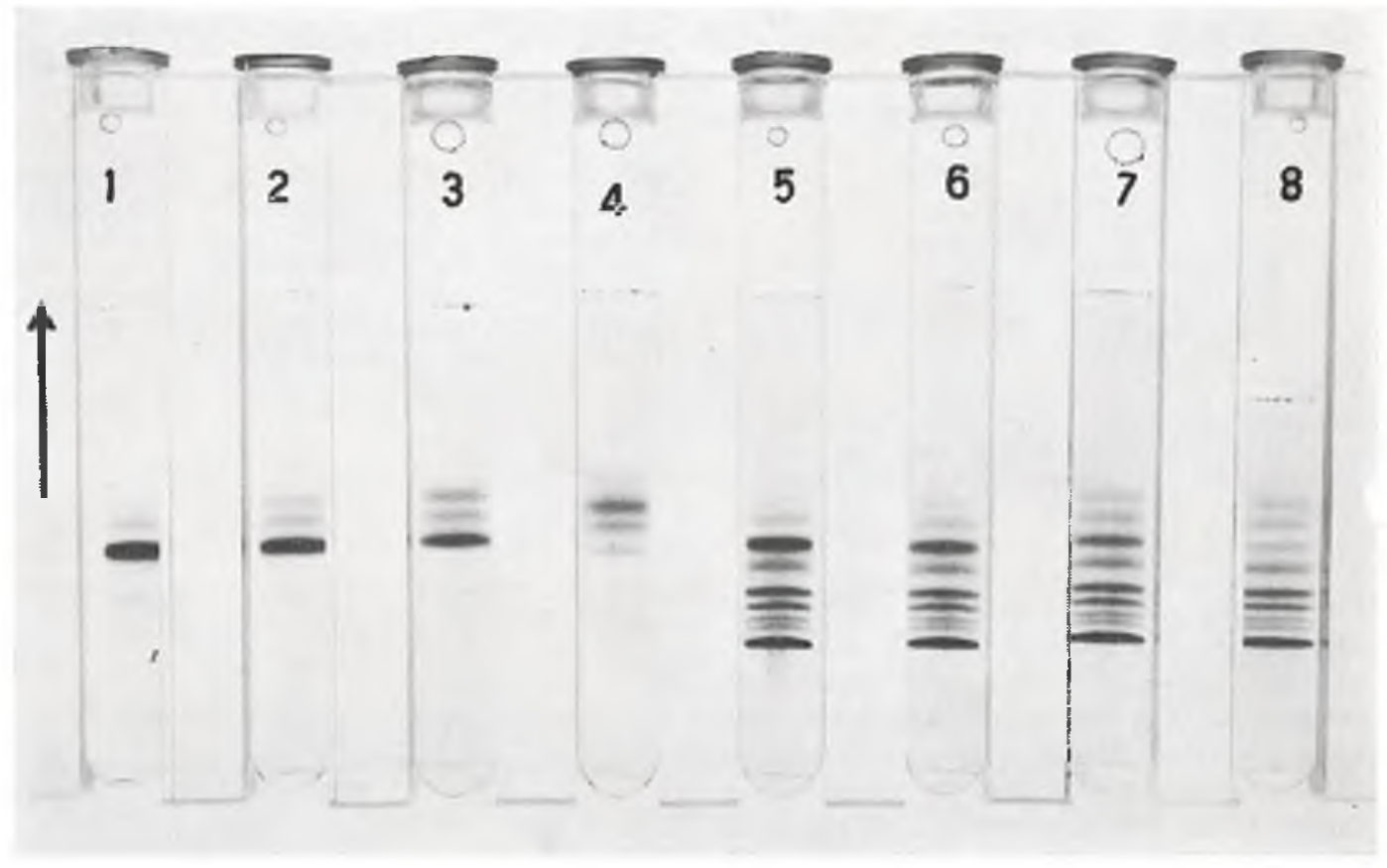

FIGURE 4

Polyacrylamide gel electrophoresis at pH 3.0 in $6 \mathrm{M}$ urea after incubation of fragment 8 with pepsin at pH 3.7 . The arrow indicates the direction of migration. The incubation time was $1,3,10$, or 30 min for the patterns $1,2,3$, and 4 respectively. After adding these samples to the peptic digest of albumin (digestion at pH 3.7 for $30 \mathrm{~min}$ ), the patterns $5,6,7$, and 8 respectively were obtained. 
TABLE 1

Amino acid content and $C$ - and N-terminal amino acids of albumin and the fragments 8,9 , and 10

The amino acid content of 8,9 , and 10 was calculated assuming that the molecular weights were respectively $15,000,12,000$, and 3,000 .

For comparison of the amino acid composition of fragment 8 with that of albumin, the amino acid composition of albumin was calculated using a molecular weight of 15,000 instead of 67,000 which is the real molecular weight.

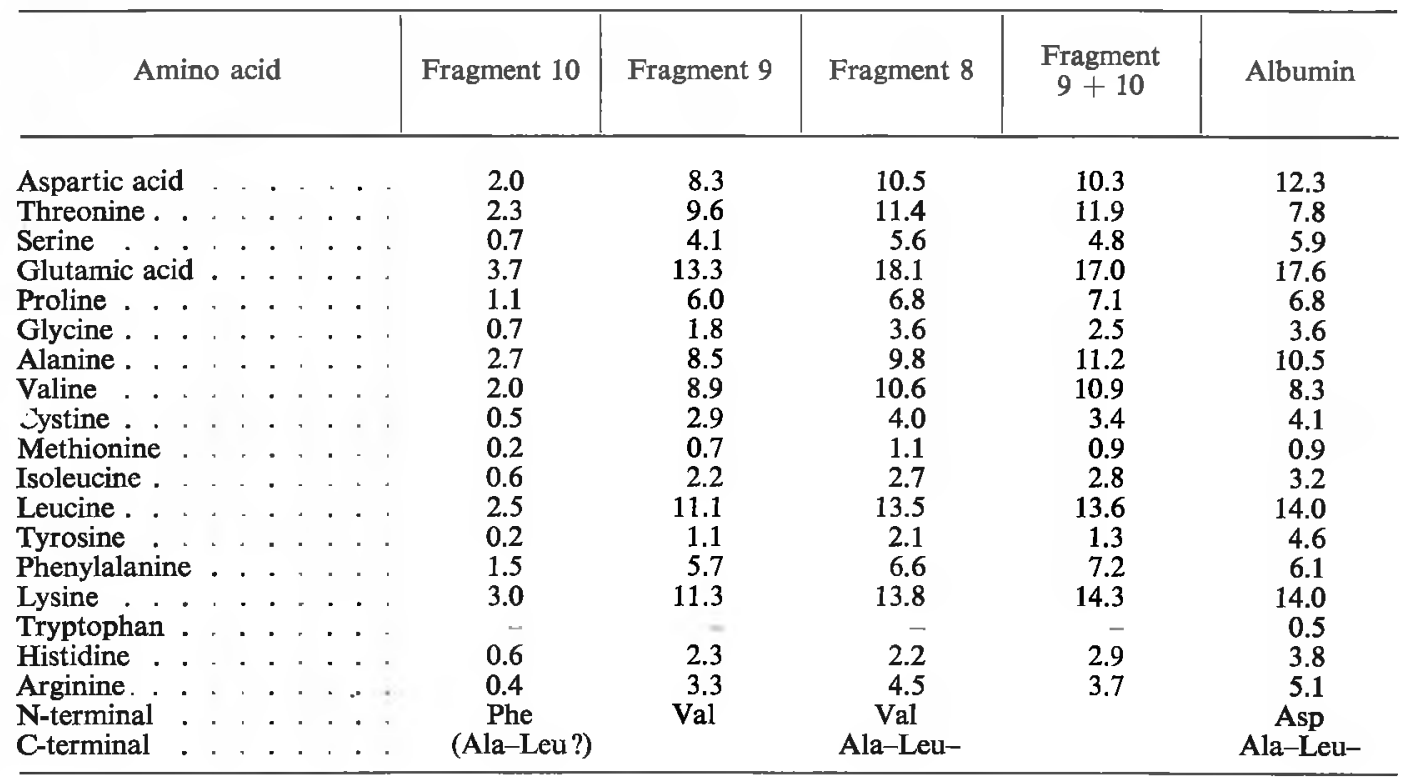

itself. So we think that this fragment is C-terminal in albumin. When fragment 10 is C-terminal placed in 8 , it must also have-Leu-Ala $\mathrm{OH}$ as C-terminal sequence. Fragment 10 appeared to be pure in respect to phenylalanine as $\mathrm{N}$ terminal amino acid. However, it was not clear whether its C-terminal sequence was -Leu-Ala $\mathrm{OH}$ or $-\mathrm{Ala}-\mathrm{Leu} \mathrm{OH}$.

It is possible that pepsin has removed a short eptide from the end of some fragments 10 . Since 9 and 10 are isolated from a peptic digest of albumin itself and not from a digest of fragment 8 alone, it cannot be fully excluded that a few other fragments with about the same size of 9 and 10 are also isolated. However, these fragments then must be very similar to 9 and 10 because 8,9 , and 10 are homogeneous in respect to polyacrylamide gel electrophoresis, gel filtration on Sephadex G-50 and G-25, and N-terminal amino acid.

Fig. 5 gives the normal and differential hydrogen ion titration curve of fragment 8 . The left peak of the differential curve is located at $Z_{I}=$ -0.6 , the right peak at $Z_{\text {II }}=-3.3$. From this distance between the two peaks a number of two titratable histidine residues can be derived assuming that the $a$-amino group is partially titrated in this region (cf.7, 8). From $Z_{I}$ the number of titratable carboxyl groups $\left(n_{c}\right)$ can be calculated using the approximate relation $\mathrm{Z}_{\mathrm{I}}=$ $\mathrm{Z}_{\mathrm{m}}-\mathrm{n}_{\mathrm{c}}$, in which $\mathrm{Z}_{\mathrm{m}}$ represents the maximum positive proton charge. From titration to low $\mathrm{pH}$, we found $Z_{m}=20 \pm 0.5$. So in first approximation, $n_{c}$ will be $20.6 \pm 0.5$. Actually a good fitting calculated curve could only be obtained with $Z_{m}=20.4$ and $n_{c}=21$. For these calculations a number of 14 titratable lysine residues was assumed. The finding of $n_{c}=21$ means that about 9 of the 30 carboxyl groups (18 Glu +11 Asp + $1 \alpha$-carboxyl) are present in the amide form. The experimentally found value of $Z_{m}$ is 20 and differs only one from the value of 21 obtained from the amino acid analysis. This difference falls within the limits of the experimental accuracy of the methods used. The titration curves of the other fragments have also been determined but are not shown here because they did not show two clearly separated peaks in the differential 


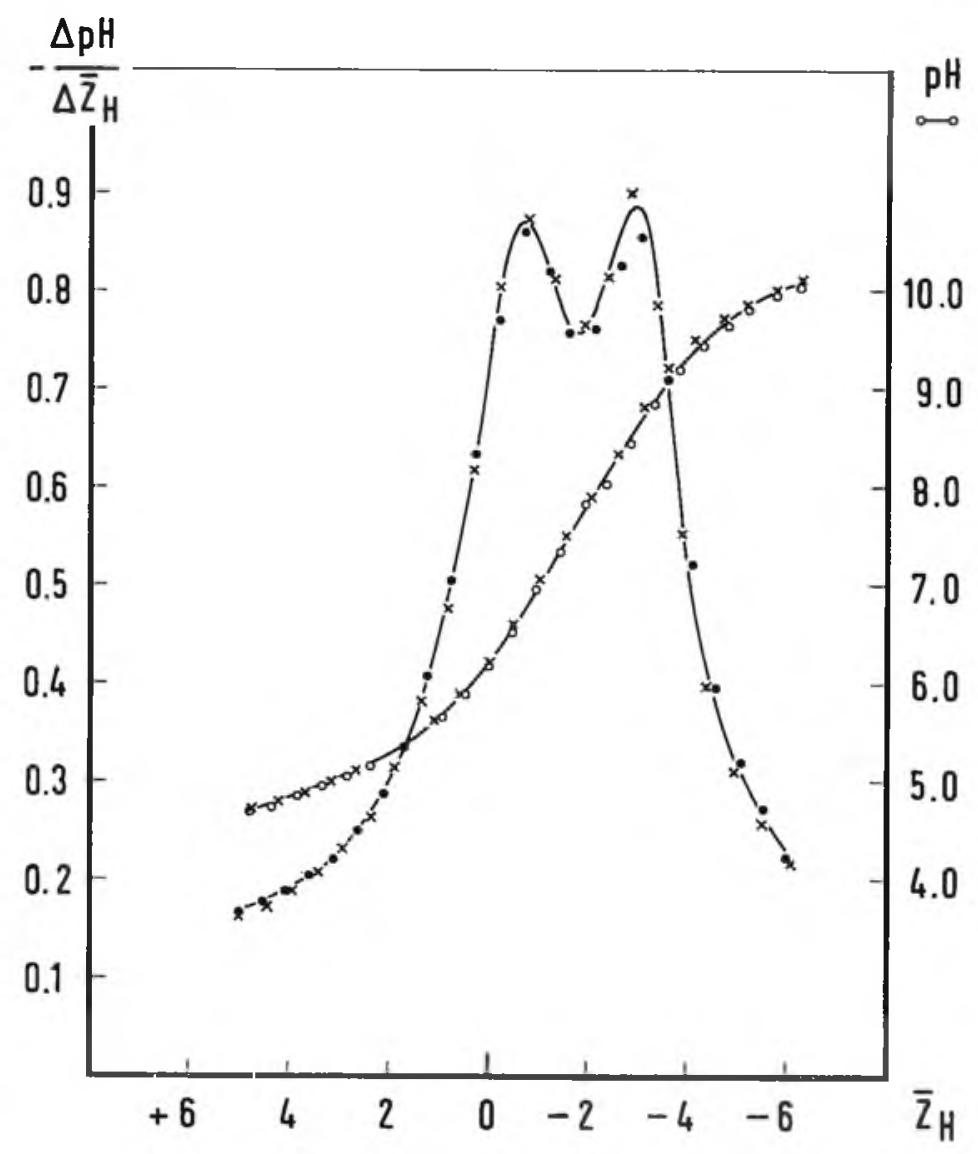

FIGURE 5

Normal and differential hydrogen ion titration curve of fragment 8.

$x$ and $O$ or $x$ and $\bullet$ refer to two different samples.

$\overline{\mathbf{Z}}_{\mathrm{H}}$ is the mean proton charge of the molecule. The molecular weight was assumed to be 15,000 . curves. Determination of the number of titratable residues then is very difficult.

Fragment 8 seems to be an interesting part of albumin. It is easy to isolate in a reproducible way. Its amino acid composition has a close re-

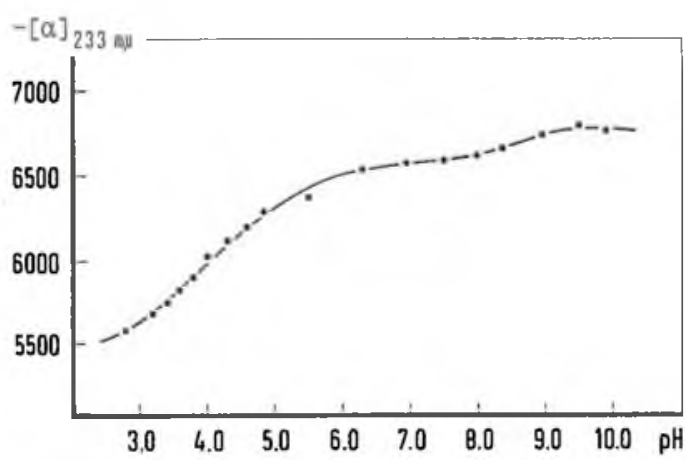

Figure 6

Specific rotation of fragment 8 at $233 \mathrm{~m} \mu$ as a function of the $\mathrm{pH}$ in $0.1 \mathrm{M} \mathrm{KCl}$. semblance to that of albumin itself (see Table 1), but the helical content is much lower. From the Moffit-Yang equation (13), the Modified Two Term Drude equation (14), and the optical rotation at $233 \mathrm{~m} \mu$ using poly-L-glutamic acid (15) or albumin (16) as reference, we found for fragment $8: 36,37,31$, and $35 \%$-helix respectivel: The molecule is fairly stable above $\mathrm{pH} 6$ with regard to its helical content (as determined by measuring the optical rotation at $233 \mathrm{~m} \mu$ ) but below pH 6 this parameter gradually decreases by about $15 \%$ (Fig. 6). This unfolding, which appears to be reversible, also becomes apparent from the analysis of the differential titration curve. The calculated curve did not fit the experimental one below pH 6, probably because the electrostatic interaction factor is not constant in this $\mathrm{pH}$ region, indicating a conformational change.

Fragment 8 can still be hydrolysed by pepsin and if we think in terms of the model of Foster (see above), it could consist of two compact 
units. However, fragment 8 does not follow the NF transition as appears from measurements of the optical rotation at $313 \mathrm{~m} \mu$ in the $\mathrm{pH}$ range of the transition $(16,17)$. This suggests that the part of the transition which can be followed by the optical rotation at $313 \mathrm{~m} \mu$ is not localized in fragment 8.

\section{ACKNOWLEDGEMENT}

We thank Mrs. C. M. G. F. Moerkerken-Van Pelt and Mr. J. J. M. Joordens for their valuable technical assistance, Dr. L. H. M. Janssen for the interpretation of the titration curves, Mr. M. G. J. Buys for carrying out the amino acid analyses, and Miss M. L. I. G. Harsveld for typing the manuscript. This work was supported in part by the Netherlands Foundation for Chemical Research (S.O.N.) with financial aid from the Netherlands Organization for the Advancement of Pure Research (Z.W.O.).

\section{REFERENCES}

1. BraAm, W. G. M., HaRmsen, B. J. M., and VAN Os, G. A. J., Biochim. Biophys. Acta, 236, 99 (1971).

2. Foster, J. F., In F. W. Putnam (Editor), The plasma proteins. Academic Press, Inc., New York, 1960, Vol. I, p. 220.
3. Weber, G., and Young, L. B., J. Biol. Chem., 239, 1415 (1964).

4. Bloemendal, H., Dissertation, Amsterdam, 1957.

5. Brenner, M., Niederweiser, A., and Pataki, G., In E. STAHL (Editor), Dünnschicht Chromatographie. Springer Verlag, Berlin, 1962, p. 433.

6. Peters, T., Logan, A. C., and Sanford, C. A., Biochim. Biophys. Acta, 30, 88 (1958).

7. De BrunN, S. H., and VAN Os, G. A. J., Rec. Trav. Chim. Pays-Bas, 87, 861 (1968).

8. J ANSSEN, L. H. M., DE BrunN, S. H., and VAN Os, G. A. J., Biochim. Biophys. Acta, 221, 214 (1970).

9. VAn Es, W. L., and Bont, W. S., Anal. Biochem., 17, 327 (1966)

10. Peters, T., JR., and Hawn, C., J. Biol. Chem., 242, 1566 (1967).

11. Sctiwert, G., J. Amer. Chem. Soc., 79, 139 (1957).

12. Braunitzer, G., Hilschmann, N., and Müller, R., Z. Physiol. Chem., 318, 284 (1960).

13. YANG, J. T., In G. D. Fasman (Editor), Poly- $\alpha-$ amino acids. Marcel Dekker, Inc., New York, 1967.

14. Shechter, E., Carver, J. P., and Blout, E. R., Proc. Nat. Acad. Sci. U.S., 51, 1029 (1964).

15. Tomimatsu, Y., Vitello, L., and Gaffield, W., Biopolymers, 4, 653 (1966).

16. SoGAMI, M., and Foster, J. F., Biochemistry, 7, 2172 (1968).

17. LeONARD, W. J., JR., and Foster, J, F., J. Biol. Chem., 236, 2662 (1961). 